\title{
Importance of Electronic Relaxation for Inter-Coulombic Decay in Aqueous Systems
}

Craig P. Schwartz, 1,2 Shervin Fatehi, $1,2,3$ Richard J. Saykally, 1,2 and David Prendergast,**

1Department of Chemistry, University of California, Berkeley, California 94720, USA

2Chemical Sciences Division, Lawrence Berkeley National Laboratory, Berkeley, California 94720, USA

3Kenneth S. Pitzer Center for Theoretical Chemistry, University of California, Berkeley, California, 94720 USA

4Molecular Foundry, Lawrence Berkeley National Laboratory, Berkeley, California, 94720 USA

Inspired by recent photoelectron spectroscopy experiments on hydroxide solutions, we have examined the conditions necessary for enhanced (and, in the case of solutions, detectable) inter-Coulombic decay (ICD) - Auger emission from an atomic site other than that originally excited. We present general guidelines, based on energetic and spatial overlap of molecular orbitals, for this enhancement of inter-Coulombic decay-based energy transfer in solutions. These guidelines indicate that this decay process should be exhibited by broad classes of biomolecules and suggest a design criterion for targeted radiooncology protocols. Our findings show that photoelectron spectroscopy cannot resolve the current hydroxide coordination controversy.

An Auger process (see Fig. 1) involves the decay of a photo-excited electron-hole pair via annihilation of the hole by another electron, with simultaneous emission of an electron from a bound state to the continuum [1]. The rate is governed (in a Fermi golden rule framework) by both direct and exchange Coulomb integrals [2]. For photo-excited holes in inner-valence or core states, the associated orbitals are well localized on a given atom, such that Auger spectra are typically dominated by atom-specific transitions. Atomic and molecular phases comprise mainly localized electronic orbitals, and the decay rate is consequently quite small for processes involving electron emission from any atomic site other than that originally excited. In such systems, this type of "off-site" emission has been labeled as ICD, inter-Coulombic (atomic or molecular) decay [3].

Although ICD has been studied primarily in the context of valence excitations [3-5], recent experiments have shown that core-excited systems may also decay in this fashion, as shown schematically in Fig. 1 [6]. Panel (a) depicts the instantaneous ground-state potential landscape and electron configuration for two atoms separated by some distance. The core electrons have energy substantially lower than the valence electrons and are tightly bound to the atomic nucleus, screening the nuclear charge $Z$ such that the valence electrons are subject to an effective nuclear Coulomb potential proportional to Zeff $1 / 4 Z_{-} 2$, consistent with Gauss's law. (For the purpose of this discussion, we assume that A and B are atoms of first-row elements with 1s core electrons only.) These effective potentials, when added together, give rise to the multiple-Coulombwell landscape shown in Fig. 1(a), which will support some localized bound states and additional bonding states spanning multiple atomic centers. Core excitation of a given atom [Fig. 1(b)] will increment the effective nuclear charge, steepening the local potential landscape at that site. This will cause a sudden downward shift in the energy of some electronic states, which in some cases may prevent coupling with states from neighboring atomic sites, or in others may lead to new electronic hybridization via tunneling through the resulting potential barrier. The key point for the present work is that the initial excitation process takes place on a time scale of tens of attoseconds, with the subsequent Auger decay taking place on a time scale of a few femtosecondslong enough for electronic relaxation to occur, but possibly too short a time for significant motion of the nuclei [6]. In Fermi's golden rule expression for the decay rate, the appropriate initial states will then correspond not to the ground-state picture in panel (a), but rather to the core-excited picture in panel (b). Once the decay takes place, with a valence electron dropping into the core hole and the excited electron emitted, as shown in panel (c), the effective potential seen by the valence electrons is restored to its initial form. Any electrons emitted subsequently will be found at significantly lower experimental energies.

Aziz et al. recently performed photoelectron spectroscopy (PES) of $\approx$ 4:0 M hydroxide solutions; sharp features were observed which the authors maintained could only be explained by invoking ICD [6]. The mechanism suggested was that ICD takes place selectively, involving neighboring waters to which the hydroxide ion donates a hydrogen bond. This interpretation is consistent with only one of the two principal models for hydroxide solvation. The "hypercoordinated" (or four-coordinate) model OH_ðH2OP 4 allows for hydrogen-bond donation during a transient change in coordination to $\mathrm{OH}_{-} \mathrm{\partial H}_{2} \mathrm{OP}$ 3, while the competing, predominantly three-coordinate model does not ever allow hydrogen-bond donation [7,8]. This hydrogenbond-based selectivity was used to explain why ICD was not observed for an isoelectronic solute, fluoride [6]. A question left unanswered is why the spectrum of neat water would not also exhibit ICD.

In order to achieve a deeper understanding of these PES experiments, we have performed detailed electronic structure calculations of hydroxide (and related molecules) solvated by water in the condensed 
phase. Specifically, we have examined the occupied states of molecules core-excited into their lowest unoccupied molecular orbitals; this excitation is identical to that in the PES experiment on hydroxide. We use the excited electron and core hole $(\mathrm{XCH})$ method, already demonstrated to work well for molecular liquids. The XCH method models the associated excited states self-consistently, with the combination of a full electronic core hole on the excited atom and an excited electron, which significantly screens the core hole in molecular systems $[9,10]$.

Our electronic structure calculations employed density solvated by water in the condensed phase. Specifically, we have examined the occupied states of molecules core-excited into their lowest unoccupied molecular orbitals; this excitation is identical to that in the PES experiment on hydroxide. We use the excited electron and core hole $(\mathrm{XCH})$ method, already demonstrated to work well for molecular liquids. The $\mathrm{XCH}$ method models the associated excited states self-consistently, with the combination of a full electronic core hole on the excited atom and an excited electron, which significantly screens the core hole in molecular systems $[9,10]$. Our electronic structure calculations employed density functional theory using the PerdewBurke-Ernzerhof (PBE) functional theory using the Perdew-Burke-Ernzerhof (PBE) form of the generalized gradient approximation to the exchange-correlation potential [11]. Although density functional theory without hybrid functionals is known to describe the hydroxide radical poorly [12], the hydroxide "radical" treated here is different in character (having two unpaired electrons), and comparisons with calculations using the PBE0 functional indicate that PBE can treat the x-ray absorption of this system satisfactorily. For excitations at the relevant $\mathrm{K}$ edge, the core-hole excited state is modeled by explicit inclusion of the 1s atomic core hole (using a modified pseudopotential) and inclusion of the excited electron occupying the lowest unoccupied molecular orbital. The atomic nuclei remain fixed in place, as they will not move large distances over the less than 4 femtoseconds before photoemission would occur [6]; no meaningful effect was observed when motions were included for hydroxide.We adopt a plane-wave representation and use a pseudopotential approximation for valence electronic structure. In all of our calculations, we use norm-conserving pseudopotentials with a numerically converged plane-wave cutoff of $85 \mathrm{Ry}$. We analyze the population of the bound states within the $\mathrm{XCH}$ approximation [10].

Condensed phase snapshots of 31 water molecules and a single $\mathrm{OH}_{-}$were provided by Professor Mark Tuckerman. Ten snapshots were analyzed of both three-and four-coordinate hydroxide [8]. For water, six snapshots were used from a plane-wave simulation of 32 water molecules, meaning a total of 192 calculations were performed for comparison. For fluoride, ten snapshots were generated by classical molecular dynamics with approximately 75 water molecules [13]. All calculated densities of states include Gaussian convolution of $0.7 \mathrm{eV}$ FWHM.

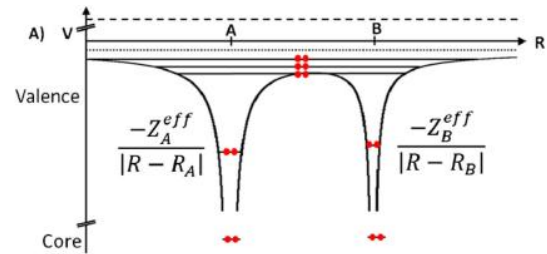

FIG.1(color online). A schematic drawing of ICD in a core-excited system. Panel (a) depicts the effective potential landscape of the valence electrons of two neighboring atomic centers as determined by the number of core electrons via Gauss's law. In panel (b), the atom at the left has been core-excited with a concomitant change in its effective valence potential. Certain states which previously were shared between both centers now are localized. In panel (c), the ICD process occurs and the effective potential for the valence electrons returns to its groundstate form. See text for more details.

core

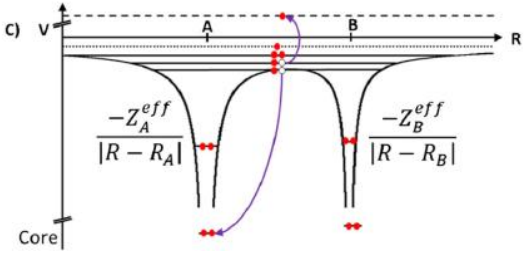

Figure 2 shows the overall valence electronic density of states (DOS) in the photo-excited state of solutions of neat water, fluoride anion in water, and hydroxide within the hypercoordinated model for both 
three- and four-coordinate hydroxide in water, with the three-coordinate ion donating a hydrogen bond. The total DOS of each solution is plotted together with the corresponding S- and p-type projected densities of states (pDOS) of the excited species, indicating the energetic overlap of its states with the surrounding solvent. The DOS includes all doubly occupied orbitals up to the top of the valence band, which defines the zero on the energy scale. We omit the DOS of the excited singly occupied orbital filled by resonant x-ray excitation. We assume that the energetic overlap indicates the possibility of spatial overlap of valence orbitals on the excited species with neighboring water molecules (evidence to follow). Because the exchange term of the Auger decay rate expression for off-site emission (referred to as ICD in [6]) is enhanced only when the corresponding decaying valence states overlap in space, we can literally see the reason for the lack of ICD in (a) water and (d) fluoride: Our calculations clearly show that the p-type pDOS of the excited molecule does not overlap significantly with the water DOS. In other words, core-excited water and fluoride do not have states capable of significant hybridization with the solvent; in terms of Fig. 1(b), every occupied state is localized in one potential well or the other.

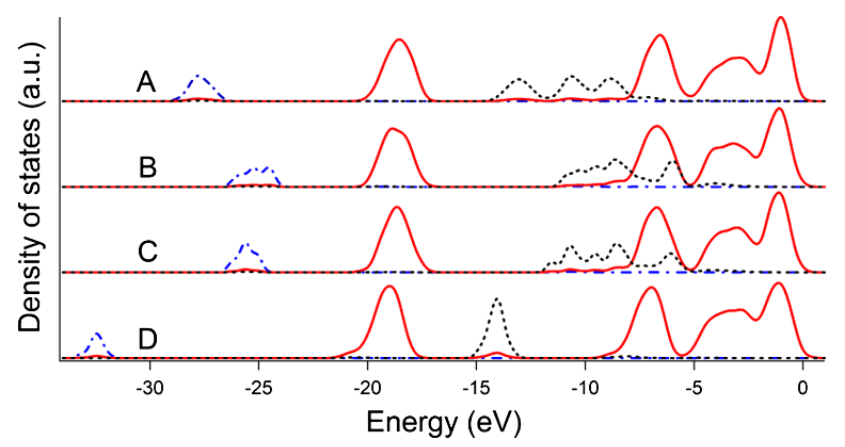

FIG. 2 (color online). The average calculated total DOS (solid solution together with the s-type pDOS (dot-dashed blue line) and p-type pDOS (dotted black line) of core-excited molecules for (a) pure water and (b) three-coordinate hydroxide, (c) four-coordinate hydroxide, and (d) fluoride solutes. Both types of pDOS in (a), (b), and (c) have been scaled to one-third the integrated intensity of the DOS; in (d), the scaling is set to one sixth. Note that the DOS for the solution does not strongly overlap with the pDOS of the coreexcited water or fluoride. Both forms of hydroxide display a feature with strong overlap at approximately $-6 \mathrm{eV}$ binding energy, independent of hydrogen-bond donation.

Hydrogen-bond donation does not appear to be a necessary condition for ICD enhancement; rather, efficient off-site energy transfer requires spatial overlap of the corresponding states of the excited and acceptor species. (Similar exchange driven enhancement of the ICD signal has been observed in weakly interacting systems by reducing intermolecular distances $[14,15]$.) In this physical picture, core-excited water and fluoride fall into gaps in the electronic density of states of the solvent. In contrast, both (b) three- and (c) fourcoordinate hydroxide ions, when core-excited, have some states that overlap in energy (and also spatially, as shown below) with those of neighboring water molecules. Consequently, energy is transferred efficiently from the ion to an acceptor water molecule, and ICD will take place.

We note that sharp features in the spectra of Aziz et al. were attributable to ICD only because photoexcited hydroxide undergoes ICD largely by way of a single valence state, as indicated by our calculations. That is why sharp peaks were obtained: Transfer will occur primarily in a narrow range of energy (largely within $1 \mathrm{eV}$ of the $\approx-6 \mathrm{eV}$ binding energy). If this band were more diffuse in energy, the resulting PES from ICD would be "blurred out" correspondingly. It is also known that the direct term in the ICD rate expression is roughly equal for hydroxide and water; the marked differences in their spectra must therefore originate in the exchange term.

These findings are illustrated in Fig. 3 for several representative doubly occupied orbitals. The excited states of the fluoride solution and of neat water are localized on the initial core-excited atoms, whereas both three- and four coordinate hydroxide states are substantially delocalized into the surrounding solvent. That this delocalization could occur at all can be seen as the overlap in energy of the total DOS for the solution and the $\mathrm{p}$-type $\mathrm{pDOS}$ of the solute. The $\mathrm{p}$ state of hydroxide is insensitive to hydrogen-bond donation and, therefore, PES must be similarly insensitive. Note that we do not calculate the PES spectrum explicitly, as has been done in other studies [16], due to the infeasibility of simultaneously performing accurate simulations of bulk properties of the disordered medium and the necessary configurational sampling.

In order for state mixing to occur, the core-excited species' valence state(s) must, to some extent, correspond energetically to those of any neighbors. The valence energy will be strongly influenced by the identity of the specific atom excited, principally through the effective nuclear charge. For example, coreexcited states of fluoride will typically have valence states shifted lower in energy than those of oxygen, which will be lower than those of nitrogen or carbon. Another factor is local electrostatics: As compared to hydroxide, a water molecule includes an additional Coulomb potential from a second proton, interaction with 
which will lower the ground-state valence energies. Additional calculations show, for instance, that states associated with core-excited nitrogen in protonated glycine, a simple amino acid, will not mix with water, while those in the deprotonated anionic species permit mixing due to a smaller downward shift in energy. Cytosine also exhibits significant mixing, implying that core-level ICD could be useful in radiooncology, particularly when carbon and nitrogen atoms are excited at their corresponding $\mathrm{K}$ edges, rather than from valence states, as discussed previously [4,5]. Sharp peaks in the PES of these systems would not be expected, however, as the energetic overlap of the valence pDOS with the solvent spans a much broader energy range than in hydroxide.

Drugs containing heavy-metal targets capable of energy transfer via ICD to cancerous DNA would be a valuable new tool in radiooncology $[4,5,17]$. The state-mixing criterion established here would allow for rational design of this type of therapeutic protocol: Core excitations in heavy metals occur at characteristic wavelengths, such that irradiation of cancerous tissue selectively bound to a heavy-metal complex with a tuned x-ray source would help to localize the radiation dose [18-20] and energy transfer, increasing the damage to cancerous tissue. There would also be a reduction in the associated damage to healthy tissue owing to its transparency to the greater portion of the secondary electrons emitted [21]. The specific nature of the impact of core excitations on valencestate electronic structure indicates the potential for careful tailoring to individual targets (such as DNA or other critical cell structures) by tuning the structure of the heavymetal complex. At the time of writing, we are actively pursuing this line of investigation in simple models of biological solutions.

In conclusion, we have outlined a generally applicable, computationally inexpensive approach to the prediction of core-excited ICD in solutions. This approach is based on an explicit treatment of electronic relaxation in the core-excited state, as approximated by the $\mathrm{XCH}$ method, and on simple criteria of energetic and spatial orbital overlap.

This work was supported by the Director, Office of Basic Energy Sciences, Office of Science, U.S. Department of Energy under Contract No. DE-AC02-05CH11231 through the LBNL Chemical Sciences Division and the Molecular Foundry, and by the National Science Foundation. Computational resources were provided by NERSC, a DOE Advanced Scientific Computing Research User Facility. We thank Professor Mark Tuckerman for the solution snapshots and Dr. Keith Lawler for helpful discussions.

This document was prepared as an account of work sponsored by the United States Government. While this document is believed to contain correct information, neither the United States Government nor any agency thereof, nor The Regents of the University of California, nor any of their employees, makes any warranty, express or implied, or assumes any legal responsibility for the accuracy, completeness, or usefulness of any information, apparatus, product, or process disclosed, or represents that its use would not infringe privately owned rights. Reference herein to any specific commercial product, process, or service by its trade name, trademark, manufacturer, or otherwise, does not necessarily constitute or imply its endorsement, recommendation, or favoring by the United States Government or any agency thereof, or The Regents of the University of California. The views and opinions of authors expressed herein do not necessarily state or reflect those of the United States Government or any agency thereof or The Regents of the University of California. 
*dgprendergast@1bl.gov

[1] H. A ${ }^{\circ}$ gren, A. Cesar, and C.-M. Liegener, Adv. Quantum

Chem. 23, 1 (1992).

[2] V. Averbukh, I. B. Mu“ ller, and L. S. Cederbaum, Phys. Rev. Lett. 93, 263002 (2004).

[3] L. S. Cederbaum, J. Zobeley, and F. Tarantelli, Phys. Rev. Lett. 79, 4778 (1997).

[4] T. Jahnke, H. Sann, T. Havermeier, K. Kreidi, C. Stuck, M. Meckel, M. Scho“ffler, N. Neumann, R. Wallauer, S. Voss, A. Czasch, O. Jagutzki, A. Malakzadeh, F. Afaneh, Th. Weber, H. Schmidt-Bo“cking, and R. Do“rner, Nature Phys. 6, 139 (2010).

[5] M. Mucke, M. Braune, S. Barth, M. Fo“ rstel, T. Lischke, V. Ulrich, T. Arion, U. Becker, A. Bradshaw, and U. Hergenhahn, Nature Phys. 6, 143 (2010).

[6] E. F. Aziz, N. Ottosson, M. Faubel, I.V. Hertel, and B. Winter, Nature (London) 455, 89 (2008).

[7] D. Asthagiri, L. R. Pratt, J. D. Kress, and M. A. Gomez, Proc. Natl. Acad. Sci. U.S.A. 101, 7229 (2004).

[8] A. Chandra, M. E. Tuckerman, and D. Marx, Phys. Rev. Lett. 99, 145901 (2007).

[9] D. Prendergast and G. Galli, Phys. Rev. Lett. 96, 215502 (2006).

[10] C. P. Schwartz, J. S. Uejio, R. J. Saykally, and D. Prendergast, J. Chem. Phys. 130, 184109 (2009).

[11] J. P. Perdew, K. Burke, and M. Ernzerhof, Phys. Rev. Lett. 77, 3865 (1996).

[12] J. VandeVondele and M. Sprik, Phys. Chem. Chem. Phys. 7, 1363 (2005).

[13] D. A. Case, T. A. Darden, T. E. Cheatham, III, C. L. Simmerling, J. Wang, R. E. Duke, R. Luo, K. M. Merz, D. A. Pearlman, M. Crowley, R. C. Walker, W. Zhang, B. Wang, S. Hayik, A. Roitberg, G. Seabra, K. F. Wong, F.

Paesani, X.Wu, S. Brozell, V. Tsui, H. Gohlke, L. Yang, C. Tan, J. Mongan, V. Hornak, G. Cui, P. Beroza, D. H.

Mathews, C. Schafmeister, W. S. Ross, and P. A. Kollman, AMBER 9, University of California, San Francisco (2006).

[14] S. Scheit, V. Averbukh, H.-D. Meyer, J. Zobeley, and L. S. Cederbaum, J. Chem. Phys. 124, 154305 (2006).

[15] Ph.V. Demekhin, Y.-C. Chiang, S. D. Stoychev, P. Kolorenč, S. Scheit, A. I. Kuleff, F. Tarantelli, and L. S.

Cederbaum, J. Chem. Phys. 131, 104303 (2009).

[16] W. Pokapanich, H. Bergersen, I. L. Bradeanu, R. R. T. Marinho, A. Lindblad, S. Legendre, A. Rosso, S. Svensson, O. Bjo“"rneholm, M. Tchaplyguine, G. O” hrwall, N.V. Kryzhevoi, and L. S. Cederbaum, J. Am. Chem. Soc. 131, 7264 2009).

[17] R.W. Howell, Int. J. Radiat. Biol. 84, 959 (2008).

[18] J. F. Hainfield, D. N. Slatkin, and H. M. Smilowitz, Phys. Med. Biol. 49, N309 (2004).

[19] S. H. Cho, Phys. Med. Biol. 50, N163 (2005).

[20] A. K. Pradhan, S. N. Nahar, M. Montenegro, Y. Yu, H. L. Zhang, C. Sur, M. Mrozik, and R. M. Pitzer, J. Phys. Chem. A 113, 12356 (2009).

[21] A. Van der Kogel and M. Joiner, Basic Clinical Radiobiology (Oxford University Press, New York, 2009), 4th ed. 